\title{
Characterizing Trends in Human Papillomavirus Vaccine Discourse on Reddit (2007-2015): An Observational Study
}

Yuki Lama ${ }^{1}$, MPH; Dian $\mathrm{Hu}^{2}$, BSc; Amelia Jamison ${ }^{3}$, MAA, MPH; Sandra Crouse Quinn ${ }^{1,3}$, PhD; David A Broniatowski ${ }^{2}, \mathrm{PhD}$

\footnotetext{
${ }^{1}$ Department of Family Science, School of Public Health, University of Maryland, College Park, MD, United States

${ }^{2}$ George Washington University, Department of Engineering Management and Systems Engineering, Washington, DC, United States

${ }^{3}$ Maryland Center for Health Equity, School of Public Health, University of Maryland, College Park, MD, United States
}

\section{Corresponding Author:}

Yuki Lama, MPH

Department of Family Science

School of Public Health

University of Maryland

4200 Valley Drive, Bldg 225

College Park, MD, 20742

United States

Phone: 13014057574

Fax: 13013149161

Email: 1amay@terpmail.umd.edu

\begin{abstract}
Background: Despite the introduction of the human papillomavirus (HPV) vaccination as a preventive measure in 2006 for cervical and other cancers, uptake rates remain suboptimal, resulting in preventable cancer mortality. Social media, widely used for information seeking, can influence users' knowledge and attitudes regarding HPV vaccination. Little is known regarding attitudes related to HPV vaccination on Reddit (a popular news aggregation site and online community), particularly related to cancer risk and sexual activity. Examining HPV vaccine-related messages on Reddit may provide insight into how HPV discussions are characterized on forums online and influence decision making related to vaccination.
\end{abstract}

Objective: We observed how the HPV vaccine is characterized on Reddit over time and by user gender. Specifically, this study aimed to determine (1) if Reddit messages are more related to cancer risks or sexual behavior and (2) what other HPV vaccine-related discussion topics appear on Reddit.

Methods: We gathered all public Reddit comments from January 2007 to September 2015. We manually annotated 400 messages to generate keywords and identify salient themes. We then measured the similarity between each comment and lists of keywords associated with sexual behavior and cancer risk using Latent Semantic Analysis (LSA). Next, we used Latent Dirichlet Allocation (LDA) to characterize remaining topics within the Reddit data.

Results: We analyzed 22,729 messages containing the strings hpv or human papillomavirus and vaccin. LSA findings show that HPV vaccine discussions are significantly more related to cancer compared with sexual behavior from 2008 to 2015 ( $P<.001)$. We did not find a significant difference between genders in discussions of cancer and sexual activity $(P>.05)$. LDA analyses demonstrated that although topics related to cancer risk and sexual activity were both frequently discussed $(16.1 \%$ and $14.5 \%$ of word tokens, respectively), the majority of online discussions featured other topics. The most frequently discussed topic was politics associated with the vaccine (17.2\%). Other topics included HPV disease and/or immunity (13.5\%), the HPV vaccine schedule (11.5\%), HPV vaccine side effects (9.7\%), hyperlinks to outside sources (9.1\%), and the risks and benefit of HPV vaccination $(8.5 \%)$.

Conclusions: Reddit discourse on HPV vaccine encompasses a broad range of topics among men and women, with HPV political debates and cancer risk making up the plurality of the discussion. Our findings demonstrated that women and men both discussed HPV, highlighting that Reddit users do not perceive HPV as an issue that only pertains to women. Given the increasing use of social media as a source of health information, these results can inform the development of targeted online health communication strategies to promote HPV vaccination to young adult users of Reddit. Analyzing online discussions on Reddit can inform health communication efforts by identifying relevant, important HPV-related topics among online communities. 
(JMIR Public Health Surveill 2019;5(1):e12480) doi: 10.2196/12480

\section{KEYWORDS}

papillomavirus infections; prevention \& control; cancer prevention; cervical cancer; HPV; vaccination; papillomavirus vaccines; immunology; administration \& dosage; social media; health communication; infodemiology

\section{Introduction}

\section{Background}

Long-lasting infections with high-risk human papillomaviruses (HPVs) can cause cancer in parts of the body where HPV infects cells, such as in the cervix, oropharynx, anus, rectum, penis, vagina, and vulva. Virtually all cervical cancers are caused by HPV. HPV vaccination acceptance is a critical public health issue as low completion rates of the vaccine series place adolescents and young adults at risk for HPV-associated infection and cancers. The multiple factors that influence vaccine acceptance have been examined on traditional mass media and social media, including the understudied platform, Reddit. This study is the first to examine HPV discourse on Reddit, which provides insight about attitudes of HPV vaccination among a unique population that may not be readily accessible in traditional data surveillance methods [1]. Our findings build upon the literature examining attitudes toward HPV vaccination online and in the media. Our goals were to assess whether discussions on Reddit reflected the competing narratives (cancer risk vs sexual promiscuity) described in the broader discourse [2-5] and, secondly, to document and describe the full range of HPV vaccine-related topics discussed on Reddit.

\section{Human Papillomavirus Vaccination Rates and Factors Affecting Uptake}

The Advisory Committee on Immunization Practices (ACIP) at the Centers for Disease Control and Prevention (CDC) recommends routine HPV vaccination for all girls and boys aged 11 and 12 years, and catch-up immunization for teens and young adults up to age 26 years, to prevent HPV infection and HPV-associated cancers [6]. The vaccine series is safe and widely effective when taken before the onset of sexual activity and potential HPV exposure. However, national vaccination rates remain low, with only $49.5 \%$ of girls and $37.5 \%$ of boys completing the recommended series [6]. Increasing vaccine uptake is a preeminent national public health concern as demonstrated by the Healthy People 2020 objective to increase HPV vaccination series completion for adolescents aged from 13 to 15 years to $80 \%$ [7].

Factors contributing to lagging vaccination rates include parental concerns about vaccine safety [8], low confidence in adolescent vaccination [9], lack of provider recommendation [10], general lack of knowledge about the vaccine [11], and concerns about adolescent sexual behavior [12,13]. In particular, as HPV is sexually transmitted, some parents fear that vaccination will give license for adolescents to engage in early or risky sexual activity [2] (this has been refuted by prior research $[2,14]$ ). Owing to the stigma associated with sexually transmitted infections (STIs), parents and providers have faced challenges addressing the sexual nature of HPV [3].
This stigma is exacerbated by the fact that the initial recommendation of HPV vaccination was for girls only. Consequently, marketing of the HPV vaccine as primarily for cervical cancer prevention in 2006 has led to continued framing of HPV as a women's health issue despite the fact that HPV affects both men and women $[15,16]$. A strong "gender bias" in vaccine uptake has been observed, as parents of male teens may feel their child is at low risk and the vaccine is unnecessary [17]. This enduring, gendered narrative has contributed to low vaccine uptake and opportunity loss for cancer prevention among men, who are increasingly affected by HPV-related anal, penile, and oropharyngeal cancers [18].

\section{Traditional and Social Media}

The media are influential in relaying HPV-related cancer risk communication and contributing to fears of adolescent sexual behavior, including promiscuity concerns. Prior content analyses of US and Canadian newspapers have found that HPV-related articles mentioning sexual promiscuity concerns ranged from $30 \%$ to $60 \%[4,5,19]$, detracting from a focus on more pertinent details of vaccination (eg, reducing HPV-associated cancers and vaccination schedule and benefits). The media's framing of the HPV vaccine as competing narratives of adolescent sexual behaviors and cancer risk may have implications for influencing public knowledge, awareness, and beliefs on HPV vaccination.

Although HPV vaccination has been studied on other media, social media may have a particularly strong impact on public perception of HPV vaccination risks. In total, $69 \%$ of adults use some form of social media [20], and research has shown that an increasing number of people seek health information online $[21,22]$. Furthermore, the open nature of social media provides a critical opportunity to investigate personal attitudes and beliefs regarding HPV vaccination. Thus, an understanding of the online discourse regarding HPV vaccination can aid public health communicators in their efforts to address public misconceptions.

Reddit is a popular online forum that allows users to post, share, and rank content through a voting system. Reddit has 542 million monthly visitors, nominating it as the fifth most visited website in the United States [23,24]. Although HPV has been studied on social media platforms such as Twitter, YouTube, and Pinterest [25-27], to our knowledge, no published study has examined Reddit content related to HPV vaccination. Reddit data capture attitudes and trends that are not readily accessible in traditional data surveillance methods, as highlighted in previous studies that used Reddit data [28,29]. Moreover, Reddit's features allow users to post anonymously, allowing users to disclose personal behaviors more readily [28,29].

Reddit's relative lack of restrictions on posting requirements and its significant number of publicly available posts related to HPV vaccination make it a critical site to assess HPV discourse among its user base. Reddit is typically associated with a male adolescent culture as $67 \%$ of users are men and $59 \%$ of users 
are between the ages of 18 and 29 years [30], providing an opportunity to communicate with a group that is eligible for vaccination, at risk of HPV-associated cancers, and otherwise hard to reach. In a survey of the Reddit community [31], 83.5\% of participants identified themselves as aged between 18 and 34 years. Of these, $80.4 \%$ identified themselves as male. Thus, an analysis of Reddit provides insight into the male perspective of HPV vaccination on social media, an understudied area in the previous literature where women have been the focus.

\section{Study Objectives}

This study was the first to utilize Reddit data to gain a better understanding of online public discussions of the HPV vaccine, including determining whether online discussions mirror previous research and media coverage. We observed how the HPV vaccine is characterized on Reddit over time and by user gender. Furthermore, to the extent that those two topics may not be representative of the total HPV-related discourse on Reddit, we conducted an additional topic analysis. Our research questions (RQs) are as follows:

\section{RQ1: Are Reddit messages more related to cancer risks or to sexual behavior?) \\ RQ2: What other topics characterize the discussion on HPV vaccination on Reddit?)}

Studying cancer risks, sexual activity, and other emerging topics from Reddit content would provide greater insight into extant online discussions about HPV vaccine, which can inform concerted, tailored health communication efforts to promote HPV vaccination and improve uptake rates.

\section{Methods}

\section{Data Source}

We used Reddit as our main data source. Unlike microblogging social media platforms such as Twitter, Reddit does not limit message length, allowing users to express their opinions in depth. Communities can be organized by different topics through "subreddits," which are forums dedicated to a specific topic such as news (/r/news) or HPV (/r/HPV). Reddit also has a strong community-based moderation culture; repetitive messages or unrelated messages will often be removed by moderators of each subreddit. Although it is possible that messages in our sample are due to malicious actors, such as bots and trolls [32], Reddit's moderation culture also helps to mitigate these concerns. Therefore, Reddit comments are a promising data source for understanding how a segment of the online community perceives the risks of HPV and the risks and benefits of the HPV vaccine. The study was determined to be research that is exempt from the Institutional Review Board at the George Washington University (IRB-180804).

\section{Data Collection}

We downloaded all recent Reddit comments from January 1, 2007 , to September 30, 2015, using a platform which allows researchers to collect and share complete Reddit datasets for research purposes [33]. At the time of analysis in 2016, the most up-to-date data were culled, resulting in our use of data from 2007 to 2015 . From the total set of all messages, we identified a subset of HPV-related messages by filtering all comments containing the text "hpv" or "human papillomavirus". From the set of hpv-related messages, we further identified a subset of vaccine-related messages by filtering messages for the string "vaccin."

\section{Data Analysis}

Our first analysis was designed to answer RQ1.

Our aim was to determine whether HPV-related messages discussed sexual behavior or cancer risk more often. First, 2 annotators (YL and $\mathrm{AJ}$ ) manually annotated 50 messages and selected an initial set of keywords associated with both topic areas. The same annotators then annotated another 350 messages, in rounds of 50 each, adjusting the keywords and refining coding techniques. We agreed upon a final set of keywords as follows: a single keyword, "cancer," was sufficient to identify messages pertaining to cancer. Messages pertaining to sexual behavior included keywords derived from the "purity" category in the Moral Foundation Dictionary [34]: "piety, pious, purity, pure, clean, sterile, sacred, chaste, saint, innocent, unclean, slut, whore, dirty, impiety, impious, profane, promiscuity, promiscuous, adulter, unchaste, sexual, sex, intercourse, coitus, lovemaking, and premarital." Using these specific keywords, annotators initially began annotating for promiscuity; however, owing to a limited number of messages, this topic was expanded to include all sexual activity related to HPV (including promiscuity). Through this iterative process, we created a codebook to identify messages as either pertaining to cancer risk, sexual activity, or other (Table 1). There was moderate agreement on messages indexing sexual behaviors, Cohen kappa $=0.62$ (95\% CI 0.54 to 0.69$)$, and high agreement on messages indexing cancer risk, kappa $=0.81$ (95\% CI 0.74 to 0.88).

Next, we employed Latent Semantic Analysis (LSA) [35], a commonly used technique in natural language processing, to measure the semantic similarity between each Reddit message and the keyword lists. Specifically, we rendered the set of all HPV-related Reddit messages into a term-document matrix and transformed this corpus into a "latent semantic space." When preparing the corpus for the LSA analysis, we removed all but the 500 most frequent unigrams. We next applied term frequency-inverse document frequency weighting to the corpus. Finally, consistent with the past literature, we retained dimensions 2 to 101 when computing the Singular Value Decomposition underlying LSA. We used the underlying LSA space to measure the cosine similarity between each Reddit post and each keyword list and calculated the average similarity score per month (see Multimedia Appendix 1).

In addition, we developed a classifier to assess the gender of the user posting each message. This tool helped us understand the overall difference between male and female user discussions related to the HPV vaccine. The classifier works by identifying gender-indicating keywords and expressions included in Reddit messages. For instance, statements such as "As a man..." or "I am a straight woman..." are used as indicators of a user's gender (Multimedia Appendix 2). In some instances, there was not enough information to identify a user's gender with confidence, so those users were classified as having an unknown gender. 
Table 1. Qualitative codebook and sample messages for Research Question 1.

\begin{tabular}{|c|c|c|}
\hline Domain & Description & Sample messages ${ }^{\mathrm{a}}$ \\
\hline Cancer risk & $\begin{array}{l}\text { Messages are related to } \mathrm{HPV}^{\mathrm{b}} \text { and relationship to cancer } \\
\text { and HPV vaccine. This includes concerns related to cancer } \\
\text { and awareness of cancer risk. Comments can be any related } \\
\text { type of cancer: cervical, throat, penile, and anal. }\end{array}$ & $\begin{array}{l}\text { "it's not just genital warts, the hpv vaccine can prevent } \\
\text { cervical cancer."; "the vaccines don't prevent all causes of } \\
\text { cervical cancer, they protect against some strains of hpv } \\
\text { which can cause cervical cancer. also, they only started } \\
\text { giving that vaccine to women over } 25 \text { recently." }\end{array}$ \\
\hline General sexual behavior & $\begin{array}{l}\text { Messages are related to HPV and sexual activity including } \\
\text { discussions of age of sexual debut, risky sexual behavior, } \\
\text { multiple sex partners, and associations with promiscuity. } \\
\text { Messages need an explicit connection to HPV. Can include } \\
\text { screening behavior if it is related to sexual activity. }\end{array}$ & $\begin{array}{l}\text { "I've only had one sexual partner in my history and we } \\
\text { were both virgins, so i'm certain i'm clear. not that hpv is } \\
\text { a death sentence or anything, but i'd rather not have to deal } \\
\text { with it." }\end{array}$ \\
\hline
\end{tabular}

${ }^{\mathrm{a}}$ Messages have been lightly edited to protect user anonymity.

${ }^{\mathrm{b}} \mathrm{HPV}$ : human papillomavirus.

We manually annotated over 100 messages to test the accuracy of the classifier. A total of 2 annotators (DH and DB) read messages identified by the classifier and determined that 43 of 50 messages were correctly classified as "male" and 45 of 50 messages were correctly classified as "female." This indicates an overall accuracy of $88 \%$ (see Multimedia Appendix 3).

We also developed a classifier to assess the age of users into 7 age categories (10 to 18,18 to 24,25 to 34,35 to 44,45 to 54 , 55 to 64 , and 65 and older) consistent with Reddit surveys [31]. When reading the gender-indicating expressions (eg, "I am 27 years old"), we observed the linguistic features related to age-indicating expressions were sometimes complicated and open to misclassification. For example, some expressions discussing time or gestational age were coded as ages, resulting in the classifier achieving $67 \%$ accuracy. Nevertheless, the current accuracy is higher than the random baseline of 7 categories (14.28\%; Multimedia Appendix 4). This classifier, which is being developed, cannot be used to reach a definite conclusion of the age groups. Instead, it can be applied to report Reddit users' demographic information for the scope of this study.

Our second analysis addressed RQ2.

To further explore the topics discussed on Reddit, we used Latent Dirichlet Allocation (LDA) [36], a Bayesian topic model, to automatically segment Reddit messages into 10 probabilistic "topics" (using unigram features; hyperparameter values were alpha=. 1 and $\eta=0.01$ ). LDA assigns each word token to one of these topics, allowing one to summarize the content of each Reddit post. After examining models with 20 and 50 topics, we determined that a 10-topic model best captured the variety of discussion subjects with minimal redundancy.

For each month, we calculated how many word tokens belonged to each topic. A total of 2 annotators (YL and AJ) read the sample messages and top word tokens of each topic to determine the theme of each LDA topic. The proportional topic weight of each year was calculated by the following equation:

proportional topic weight of Topic $\mathrm{X}=$ (The frequency of all words that belongs to Topic $\mathrm{X}) /($ The total frequency of all words in given years)

The detailed results and interpretation are reported below.

\section{Results}

Research Question 1: Cancer Risk and Sexual Activity

Our final dataset included 22,729 messages filtered to include "hpv" or "human papillomavirus" and "vaccin". Mitigating concerns about behavior by malicious actors, such as bots, we found that no two messages in our dataset are exactly the same, a common indicator of bot behavior [37]. The number of messages varied by year and increased over time, from 162 messages in 2007 to a peak of 5311 messages in 2015 (Figure 1). Among those posting HPV vaccine messages $(n=12,952)$, $53.0 \%(6869 / 12,952)$ were male, $8.8 \%(1143 / 12,952)$ were female, and $38.1 \%(4940 / 12,952)$ were of unknown gender (Table 2). Nearly $17 \%$ of users were aged from 25 to 34 years, followed by $11.2 \%$ of users aged 18 to 24 years, although $60.0 \%$ of users did not specify their age (Table 2).

In manual annotation, it was clear that messages describing cancer risk were more identifiable than messages discussing HPV-related sexual behavior. Cancer risk messages tended to focus on specific themes, including the relationship between HPV infection and cancer incidence (especially related to specific HPV strains), and on HPV vaccination as a resource to prevent future cancer incidence. Messages about sexual behaviors were more varied. Initially, we attempted to code for instances strictly related to HPV vaccine and sexual promiscuity but found very few instances ( $<5$ in the first 100 messages) that we were unable to do so. The widespread use of sarcasm and irony on Reddit also made it difficult to know the meaning of some messages out of context. Many of the messages we encountered incorporated the moral foundation keywords, but only to refute the idea that HPV vaccine promotes promiscuity. To capture the complexity of this topic, we broadened our coding scheme to include any discussion of sexual activity and HPV vaccination and were able to identify a wider range of subjects including safe sex practices, personal stories, and debates over sexual risk. 
Figure 1. Number of Human papillomavirus (HPV) vaccine messages by year.

\section{HPV vaccine message frequency by year (2007-2015)}

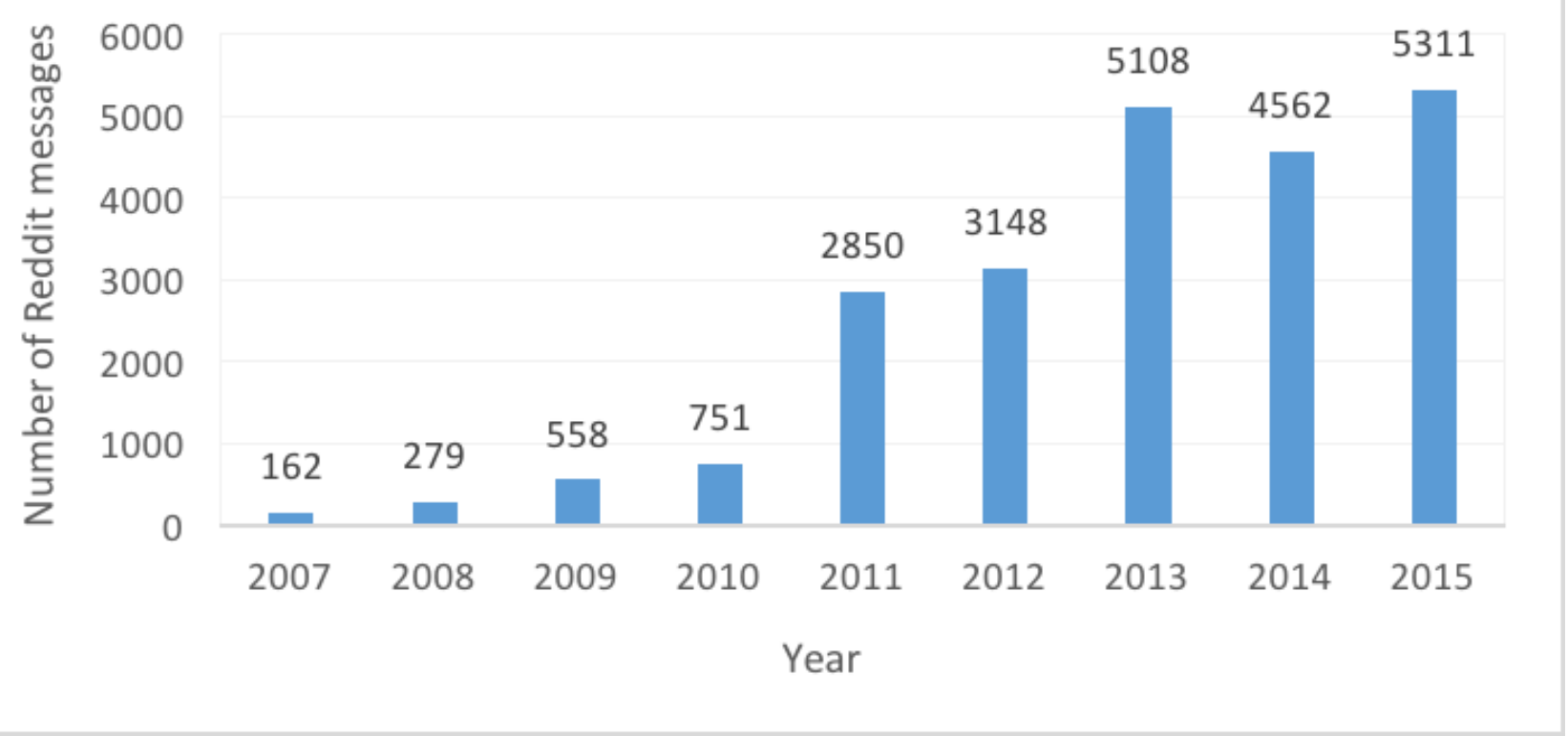

Table 2. Age and gender distribution of the Reddit sample data.

\begin{tabular}{|c|c|c|c|c|}
\hline Age group (years) & Female users & Male users & Unspecified gender & Age subtotal \\
\hline 10 to 18 & 128 & 339 & 57 & 524 \\
\hline 18 to 24 & 293 & 862 & 291 & 1446 \\
\hline 25 to 34 & 376 & 1489 & 297 & 2162 \\
\hline 35 to 44 & 103 & 549 & 65 & 717 \\
\hline 45 to 54 & 42 & 195 & 26 & 263 \\
\hline 55 to 64 & 7 & 37 & 2 & 46 \\
\hline 65 and above & 7 & 13 & 0 & 20 \\
\hline Unspecified age & 187 & 3385 & 4202 & 7774 \\
\hline Gender subtotal & 1143 & 6869 & 4940 & 12,952 \\
\hline
\end{tabular}

The LSA analysis showed that cancer is significantly more discussed than sexual activity (Table 3). Discussions about sexuality and cancer did not typically co-occur: The cosine similarity score between the cancer risk and sexual activity keyword lists in the semantic space was -0.01 , indicating that the 2 lists were essentially orthogonal. Discussions about HPV vaccine were more similar to the keyword "cancer" than to the sexual purity keywords, particularly after 2007 (Figure 2). However, the average monthly Reddit message discussed neither cancer nor sexuality (cosine similarity values were 0.11 and 0.08 , respectively). These low similarity scores indicate that other topics must exist in the corpus that are neither about cancer nor sexual behavior. Furthermore, we observed a decrease in average similarity to both cancer and sexuality over time, indicating that other topics of discourse may have emerged within these forums.

In general, we did not notice a significant difference between genders in discussions of cancer and sexual activity (all $P$ values were greater than .05 , except in 2012; note that we would expect one $P$ value to be less than .05 owing to chance alone; Figure 2). 
Table 3. $t$ test of similarity of messages between human papillomavirus (HPV) cancer risk and sexual activity on Reddit by Latent Semantic Analysis (LSA).

\begin{tabular}{lllll}
\hline Year & Message count & Sexual similarity, mean & Cancer similarity, mean & Yearly $t$ test score \\
\hline 2007 & 162 & 0.10 & 0.14 & -1.70 \\
2008 & 279 & 0.08 & 0.15 & $-4.83^{\mathrm{a}}$ \\
2009 & 558 & 0.07 & 0.13 & $-6.84^{\mathrm{a}}$ \\
2010 & 751 & 0.06 & 0.13 & $-9.26^{\mathrm{a}}$ \\
2011 & 2850 & 0.06 & 0.11 & $-11.97^{\mathrm{a}}$ \\
2012 & 3148 & 0.07 & 0.10 & $-9.35^{\mathrm{a}}$ \\
2013 & 5108 & 0.06 & 0.11 & $-19.20^{\mathrm{a}}$ \\
2014 & 4562 & 0.07 & 0.10 & $-10.25^{\mathrm{a}}$ \\
2015 & 5311 & 0.06 & 0.10 & $-14.64^{\mathrm{a}}$ \\
\hline
\end{tabular}

${ }^{\mathrm{a}} P<.001$.

Figure 2. Comparison of message similarity between human papillomavirus (HPV) cancer risk and sexual activity on Reddit by Latent Semantic Analysis (LSA).

\section{Mean of similarity score comparisons}

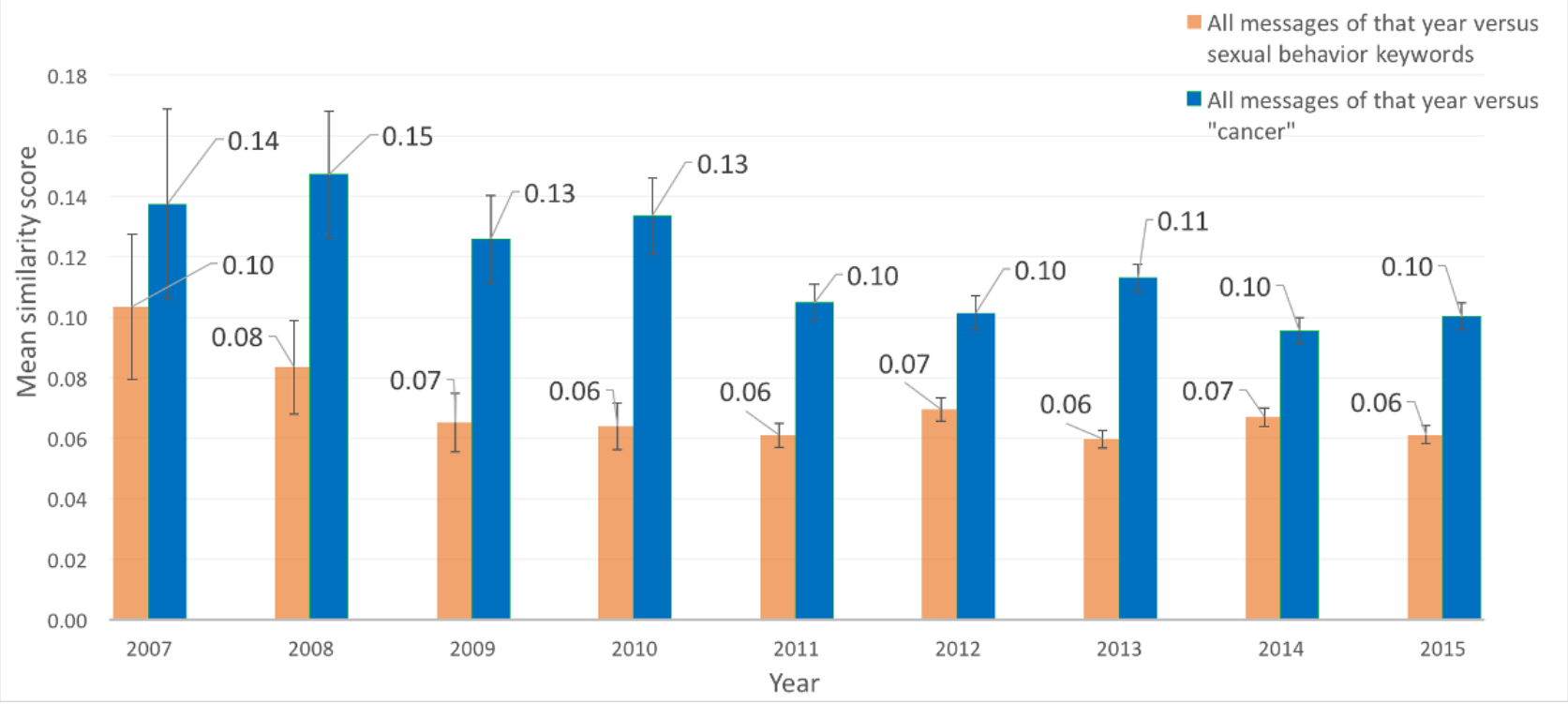

\section{Research Question 2: Selected Topic Analysis}

In our close reading of messages for manual annotation, we recognized that although messages included keywords related to cancer risk or sexual activity, there were additional topics that extended beyond the scope of either topic (Table 4). Some of these conversations were narrowly focused on HPV vaccination, for instance, debates over whether vaccinating the entire population was a cost-effective prevention method or discussions of vaccine efficacy related to particular strains of HPV. Other conversations were focused on sexual activity and health, in general. We observed a subset of conversations that detailed personal experiences with STIs and described the various steps individuals had taken to avoid all types of STIs (eg, condom use, HPV vaccination, and regular STI testing).
Another major topic was related to circumcision, as some users felt that primary prevention measures such as HPV vaccination reduced the need to circumcise (as circumcision is often cited as a method to reduce STI infection). Finally, another subset of conversations was focused on broader political and philosophical discussions that sometimes used HPV vaccination as a talking point. For instance, political discussions of the role of government sometimes questioned vaccine mandates as infringing on parental rights as part of a larger justification for libertarianism. Alternatively, users would discuss sexual education, access to contraceptives, and HPV vaccination as talking points in discussions of abortion rights (Table 4). Although these conversations were not specifically focused on HPV vaccination, we believed they reflect the broader discourse on the topic and were an important area of study. 
Table 4. Summary of human papillomavirus (HPV) vaccine topics generated by the Latent Dirichlet Allocation (LDA) approach.

\begin{tabular}{|c|c|c|}
\hline Topic name & Topic keywords & Description \\
\hline General vaccine debate (Topic 0 ) & $\begin{array}{l}\text { peopl, immun, diseas, get, like, flu, one, } \\
\text { hpv, caus, children }\end{array}$ & $\begin{array}{l}\text { Broadly concerned with vaccine decision making; Vaccine } \\
\text { safety; Risks of side effects; Importance of vaccination; } \\
\text { Personal stories }\end{array}$ \\
\hline $\begin{array}{l}\mathrm{HPV}^{\mathrm{a}} \text {-related cancer and genital warts } \\
\text { (Topic 1) }\end{array}$ & $\begin{array}{l}\text { hpv, strain, cancer, caus, wart, protect, type, } \\
\text { infect, genit, genit-wart }\end{array}$ & $\begin{array}{l}\text { HPV strains related to cancer and genital warts; Strains } \\
\text { covered by vaccine }\end{array}$ \\
\hline Sharing government links (Topic 2) & $\begin{array}{l}\text { http, www, http-www, gov, cdc, cdc-gov, } \\
\text { nih, www-cdc, nih-gov, nlm }\end{array}$ & $\begin{array}{l}\text { Vaccine concerns corroborated by links to } \mathrm{CDC}^{\mathrm{b}} \text { and } \\
\text { PubMed research articles }\end{array}$ \\
\hline HPV cancer prevention (Topic 3) & $\begin{array}{l}\text { cancer, cervic, cervic-cancer, hpv, get, } \\
\text { women, prevent, caus, hpv-vaccin, men }\end{array}$ & $\begin{array}{l}\text { Gender-related vaccine and HPV concerns; cervical cancer; } \\
\text { other HPV related cancers; recommendations for men and } \\
\text { women }\end{array}$ \\
\hline HPV-specific vaccine debate (Topic 4) & $\begin{array}{l}\text { effect, gardasil, studi, report, gt, hpv, death, } \\
\text { year, side, hpv-vaccin }\end{array}$ & $\begin{array}{l}\text { Adverse events linked to HPV vaccination; Side effect risk; } \\
\text { Vaccine efficacy; Debating facts (fewer personal stories } \\
\text { than topic 0). }\end{array}$ \\
\hline HPV vaccine political debate (Topic 5) & $\begin{array}{l}\text { peopl, hpv, make, gt, would, like, hpv-vac- } \\
\text { cin, think, one thing }\end{array}$ & $\begin{array}{l}\text { Parental vaccine rights; Reach of federal power; Abortion } \\
\text { debate; Sexual education policy; General political debate; } \\
\text { Individual politicians; Corruption }\end{array}$ \\
\hline Sharing general links on HPV (Topic 6) & $\begin{array}{l}\text { http, com, www, http-www, org, amp, en, } \\
\text { hpv, comment, wikipedia }\end{array}$ & $\begin{array}{l}\text { HPV concerns with links to news stories; Links to other } \\
\text { Reddit threads; Generally sharing single links rather than } \\
\text { a list of links (Topic 2) }\end{array}$ \\
\hline HPV vaccination schedule (Topic 7) & $\begin{array}{l}\text { get, hpv, hpv-vaccin, year, got, doctor, go, } \\
\text { shot, age, girl }\end{array}$ & $\begin{array}{l}\text { Age of vaccination; Preventive screenings; Medical advice; } \\
\text { Personal stories; Deciding whether to vaccinate }\end{array}$ \\
\hline Circumcision debate (Topic 8) & $\begin{array}{l}\text { circumcis, men, gt, risk, women, male, hiv, } \\
\text { infect, hpv, benefit }\end{array}$ & $\begin{array}{l}\text { Risks and benefits of circumcision; Likelihood of contract- } \\
\text { ing STI } \text {; Cancer prevention }\end{array}$ \\
\hline Sexual behaviors including HPV (Topic 9) & $\begin{array}{l}\text { get, hpv, sex, test, condom, peopl, like, } \\
\text { know, partner, std }\end{array}$ & $\begin{array}{l}\text { Discussions of sexual behaviors; STI prevention; Vaccina- } \\
\text { tion; Condom use; Relationship norms }\end{array}$ \\
\hline
\end{tabular}

${ }^{\mathrm{a}} \mathrm{HPV}$ : human papillomavirus.

${ }^{\mathrm{b}} \mathrm{CDC}$ : Centers for Disease Control and Prevention.

${ }^{\mathrm{c}} \mathrm{STI}$ : sexually transmitted infection.

In addition to the diversity in topics, it appears users were turning to Reddit for a variety of reasons: some were posing questions and were looking for answers; others seemed to want to debate; some just wanted to discuss topical issues in the news; and still others seemed to want to make jokes. Messages ranged from one-word responses to posts a few thousand words long. Some were based on personal experiences and anecdotes, and others were based on facts and statistics, often linking to cited research. The understanding gained from reading HPV vaccine-related messages led us to our second research question, as we realized we needed to explore the full range of topics that were being discussed on Reddit.

Expanding upon the LSA findings, the LDA topics (Table 4) generated a more comprehensive list of all HPV vaccine-related topics of interest. The most widely discussed topic was HPV-related political debate $(17.2 \%)$, wherein users posted messages on political topics including vaccine policy, abortion rights, and sex education policy. Cancer was the second most widely discussed $(16.1 \%)$, after combining the two closely related topics of HPV strains causing cancer and genital warts and HPV cancer prevention. This was followed by sexual activity and preventive behaviors (14.5\%), which focused on behaviors such as using condoms, receiving HPV vaccination, and regular testing and screenings for STIs. The fourth most popular topic was HPV disease and immunity (13.5\%), which included broad concerns with diseases and infections associated with HPV. The fifth was the vaccine schedule related to HPV $(11.5 \%)$, which included messages on age of vaccination initiation and requesting information on vaccination from physicians. The sixth, vaccine side effects and risks $(9.7 \%)$ included messages on adverse events linked to HPV vaccination. The seventh topic was related to messages that included use of government or research (eg, CDC and PubMed) papers and general websites (eg, Wikipedia and news sites) to corroborate user opinions $(9.1 \%)$. The final topic discussed circumcision, particularly the risks and benefits as related to STI and cancer prevention (8.5\%) (Figure 3). 
Figure 3. Human papillomavirus (HPV) vaccine topic distribution on Reddit.

\section{HPV vaccine topic distribution on Reddit (2007-2015)}

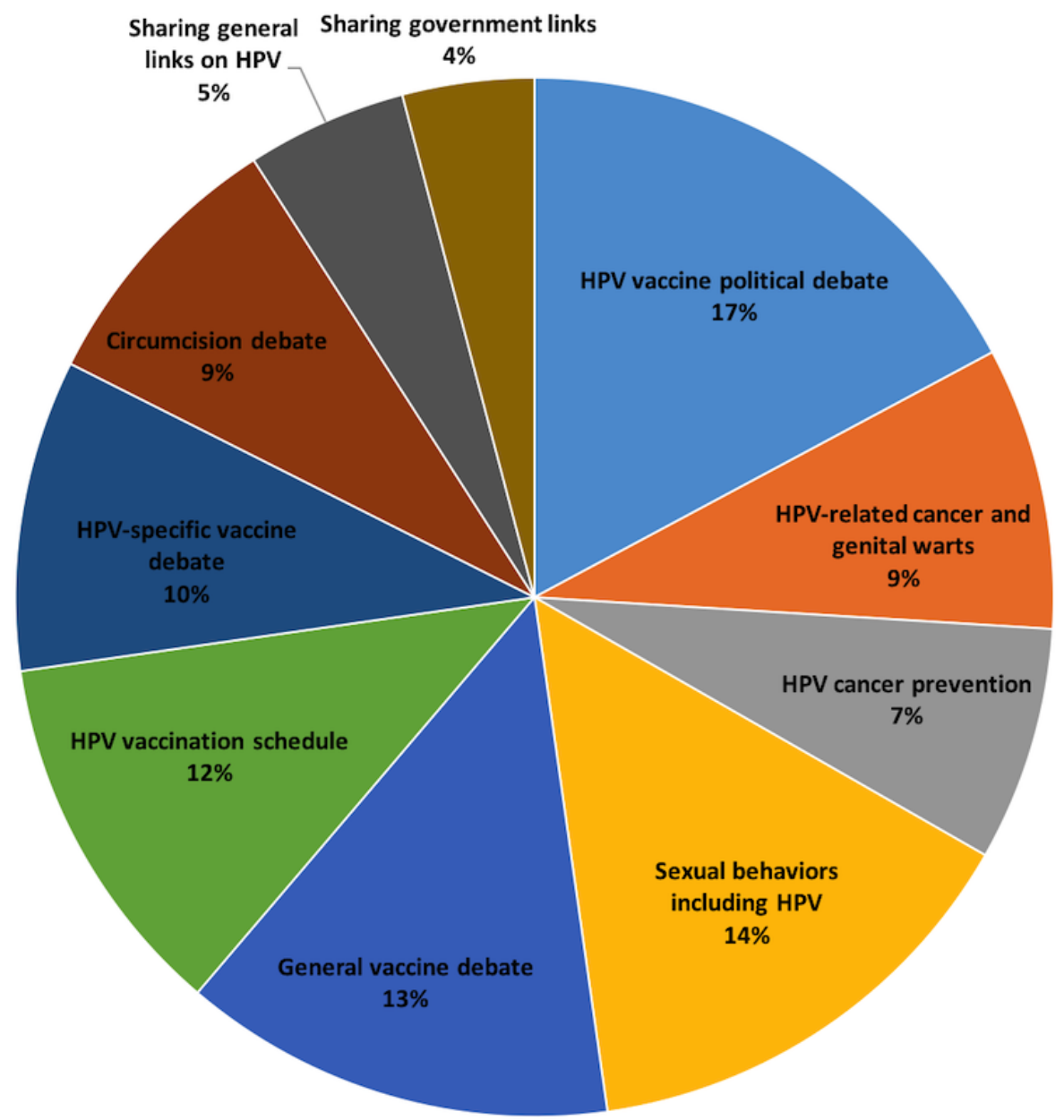

The political debate surrounding HPV vaccines peaked in 2011 (24\%) and decreased in $2013(14 \%)$, when the number of messages plateaued (Multimedia Appendix 5). General HPV vaccine messages peaked in 2008 (20\%), whereas general vaccine messages sharply decreased during 2008 (8\%) and increased in 2009 (22\%). Discussion of sexual behavior sharply increased in 2010 (18\%) and 2014 (17\%). Circumcision debate sharply increased in 2012 (11\%; Multimedia Appendix 5).

Across the LDA topics, notable spikes were apparent in certain time periods (ie, 2008, 2009, 2011, 2013, and 2014). We observed peaks for certain years across LDA topics (Multimedia Appendix 5). Specifically, we observed an increase in the discussion related to HPV-associated cancers and genital warts in 2008 (10\%; Multimedia Appendix 5). Furthermore, increases were observed across HPV cancer (11\% and 0.092) and sexual activity discussions (20\% and $18 \%$, respectively) in 2010 (Multimedia Appendix 5). Discussions of cancer increased in 2013 (8\% and 10\%, respectively) and 2014 (17\%).

\section{Discussion}

\section{Principal Findings}

Our findings emphasize increased discussion of HPV-related cancer risks on Reddit, in contrast with the mainstream media's focus on sexual behavior linked to HPV vaccine. Of the total set of HPV vaccine-related Reddit messages, $16.1 \%$ were focused on cancer risk topics and $14.5 \%$ were focused on HPV related to sexual activity. However, even when combined, these two topics represented less than one-third of the total HPV vaccine-related messages on Reddit. LSA findings did not show significant gender differences in discussions of HPV-related sexual behaviors and cancer risks, though discussions of both topics decreased over time. We identified additional topics that provide insight into the broader HPV discourse on Reddit, which included HPV disease and immunity, vaccine schedule, side effects, risks and benefits of vaccination, and the circumcision debate (Figure 3), providing further public health surveillance of nuanced HPV attitudes on Reddit.

Our LSA findings demonstrated that, on Reddit, cancer is significantly more discussed than sexual activity, consistent with the majority of prior research $[14,38,39]$. Although HPV 
cancer risk prevention and HPV-related sexuality are presented as competing narratives in the broader media discourse, these two topics alone do not reflect the focus of the majority of HPV-related conversations on Reddit. Indeed, the political debate regarding vaccination was the most discussed topic on Reddit. The HPV vaccine has been the subject of political discussion since its introduction in 2006 [40]. Messages spiked in 2008 for the HPV vaccine debate, perhaps associated with media attention surrounding state consideration for school vaccination mandates for middle-school age girls [41]. The following year also saw spikes in messages for general vaccine debate, perhaps associated with the introduction of a second HPV vaccine, Cervarix, in 2009. That same year, Gardasil was approved for boys aged 9 to 26 years to reduce genital warts; however, the ACIP did not recommend routine vaccination among males until 2011 [40]. We observed continued increases in discussion through 2011 and 2012, most likely due to CDC HPV vaccine recommendations for boys in fall 2011 coinciding with the 2012 presidential campaign, which incited debates around vaccination [40]. Increases in cancer discussions during 2013 and sexual activity in 2014 do not coincide with major policy changes or announcements and thus warrant additional research on factors that contributed to these trends.

Our analyses did not support the idea of HPV as primarily a woman's health issue on Reddit, highlighting the differences between narratives on Reddit and general public discourse. This discrepancy is surprising, given the initial recommendation and marketing of the vaccine toward girls to prevent cervical cancer [15] and given the majority of Reddit users are adolescent males. Furthermore, until recently, media coverage of HPV vaccine has largely been focused on women, particularly regarding promiscuity [42] even with expanded vaccine recommendations to include males. Our LDA findings also challenge the notion that HPV is perceived as an issue affecting women exclusively, with sizable discussion on Reddit on HPV prevention for men related to circumcision. The circumcision debate indicates broader concerns of men concerned with STI and cancer prevention related to HPV. These results further emphasize the difference between discussions on Reddit and media representations on the HPV vaccine.

The implications of inconsistencies between HPV vaccination discussion on Reddit compared with media outlets are significant. Continued media coverage of controversies may detract from focus on benefits of vaccination, which may translate to lower rates of vaccine uptake and increased incidence of cancer [43]. Public health agencies can utilize traditional and digital media to share targeted and tailored health messages promoting HPV vaccination to the broader population. Our findings demonstrated a wide spectrum of viewpoints, ranging from evidence-based posts to subjective personal experience posts with varying accuracy. Users reported questions about HPV prevention strategies (eg, vaccination vs using condoms), best practices for regular screenings (eg, Pap tests), and the benefits of vaccination while considering potential side effects. By highlighting the topics most salient to Reddit users, public health communication efforts can be targeted to suit the needs of this online community. In addition, the range and extent of messages from users seeking information highlights a gap in accessible credible information online. These messages on Reddit can pose a challenge for a user to navigate and make an informed decision regarding vaccination.

Critical to vaccine uptake is the receipt of health care provider (HCP) recommendation [42], which may increase awareness of the benefits of HPV vaccine as well as cancer risks of nonvaccination. Despite recommendations being a key driver in uptake, physicians are more likely to recommend other vaccines (ie, tetanus and diphtheria) compared with HPV vaccine [44]. Lacking HCP recommendations or accurate knowledge, users may turn to online forums such as Reddit seeking advice, which may be filled with inaccurate information. In addition, without provider and public health engagement in these forums, misconceptions surrounding HPV vaccination may proliferate, fueling vaccine hesitancy.

Our analysis of Reddit posts can inform health communication efforts tailored to users' queries. Topics such as HPV vaccination as cancer prevention can be promoted whereas other topics, such as fear of vaccine side effects, can be combated with accurate messaging. Our findings suggested an increasing interest in HPV vaccination on Reddit over time, indicating a need for public health communication through social media platforms such as Reddit. Public health officials can develop health communication strategies that engage users, such as answering questions through Ask Me Anything events on Reddit or enlisting experts or well-known public figures to promote vaccination. Providers can share accurate HPV information and engage with patients regarding their questions and concerns regarding HPV vaccination, particularly through national campaigns and events. Massey et al found that HCPs were less likely to utilize hashtags and engage in Twitter chat events, resulting in decreased online presence and missed opportunities to productively engage with users [45].

Given the increasing number of people using social media and seeking health information online, public health practitioners and agencies need to take advantage of these opportunities to connect with users online to advocate for accurate, timely information. This may help to combat misconceptions related to HPV vaccination behavior. For example, in posts related to the vaccine schedule, users questioned the need for age cutoffs for HPV vaccines, a question which can be addressed with clear messaging about eligibility, especially given the recent Food and Drug Administration approval for expanded use of individuals aged from 27 to 45 years [46]. The continued failure to address these communication needs may contribute to increased vaccine hesitancy, further decreasing vaccination rates and, subsequently, increasing HPV-related morbidities. Although the increased effort of targeted engagement with users online will require more resources (ie, dedicated time) from public health agencies, the opportunities to reach and impact HPV vaccination greatly outweigh the challenges.

\section{Limitations}

The messages are drawn from Reddit users and do not necessarily represent the range of attitudes and beliefs across the broader population. Consistent with Barthel et al, our dataset revealed that the majority of users who posted HPV vaccine messages were males $(53 \%)$ compared with females $(8.8 \%)$ 
[30]. However, the fact that Reddit users skew younger in age, with $59 \%$ users between the ages of 18 and 29 years, is in line with target audiences for vaccination, particularly at the catch-up stage (aged 18 to 26 years) [30]. Our findings do not examine other demographic variables besides binary gender (ie, male or female) and only examined messages from 2007 to 2015. During this period, we noticed spikes in conversation that did not coincide with major policy recommendations or noteworthy events, warranting a further examination of underlying causes of these upticks in discussion.

Future studies would benefit by examining other demographic variables across further time points. Although our data limited us to examine only gender and age, future research can be expanded to parse messages by user characteristics such as age and geographical area to examine complex HPV attitudes with another level of granularity. This is especially important given the disparities in HPV vaccine uptake in rural areas compared with urban areas. Furthermore, sentiment analysis may provide insight regarding differences in message content (positive, neutral, and negative) across users. Topic analysis can also be applied across social media to examine any differences in discussion across different platforms.

\section{Conclusions}

This study provided critical insight about HPV vaccination discourse on Reddit over time. In particular, we found Reddit users were discussing a wider variety of topics, beyond cancer risks and sexual activity, in contrast to prevailing media focus. Reddit can be a surveillance tool to examine emerging trends among users. In response, health communication stakeholders can utilize this platform to address concerns of Web-based communities to the extent that they are representative of the broader public, instead of concerns of the news cycle. Finally, our results indicate that HPV is discussed among both men and women on Reddit, and it is heartening that HPV is not perceived as an issue that only pertains to women. These findings may inform the development of strategies to address HPV vaccine information, while dispelling misinformation and misconceptions, to increase vaccination and promote sexual health online. As young adults increasingly use Web-based resources to seek health information, public health communication efforts are needed to leverage this opportunity to deliver timely, accurate health information to internet communities.

\section{Acknowledgments}

This research was supported by the National Institute of General Medical Sciences, National Institutes of Health (NIH; award 5R01GM114771). The content is solely the responsibility of the authors and does not necessarily represent the official views of NIH.

\section{Conflicts of Interest}

None declared.

\section{Multimedia Appendix 1}

Latent Semantic Analysis (LSA) similarity results.

[XLSX File (Microsoft Excel File), 51KB-Multimedia Appendix 1]

\section{Multimedia Appendix 2}

Gender classifier accuracy assessment.

[XLSX File (Microsoft Excel File), 34KB-Multimedia Appendix 2]

\section{Multimedia Appendix 3}

Development strategy of the gender classifier.

[DOCX File, 58KB-Multimedia Appendix 3]

\section{Multimedia Appendix 4}

Annotation to assess accuracy age classifier.

[XLSX File (Microsoft Excel File), 20KB-Multimedia Appendix 4]

\section{Multimedia Appendix 5}

Latent Dirichlet allocation (LDA) results.

[XLSX File (Microsoft Excel File), 82KB-Multimedia Appendix 5]

\section{References}


1. Dredze M, Broniatowski DA, Smith MC, Hilyard KM. Understanding vaccine refusal: why we need social media now. Am J Prev Med 2016 Apr;50(4):550-552 [FREE Full text] [doi: 10.1016/j.amepre.2015.10.002] [Medline: 26655067]

2. Forster A, Wardle J, Stephenson J, Waller J. Passport to promiscuity or lifesaver: press coverage of HPV vaccination and risky sexual behavior. J Health Commun 2010 Mar;15(2):205-217. [doi: 10.1080/10810730903528066] [Medline: 20390987]

3. Friedman AL, Shepeard H. Exploring the knowledge, attitudes, beliefs, and communication preferences of the general public regarding HPV: findings from CDC focus group research and implications for practice. Health Educ Behav 2007 Jun;34(3):471-485. [doi: 10.1177/1090198106292022] [Medline: 17000622]

4. Quintero Johnson J, Sionean C, Scott A. Exploring the presentation of news information about the HPV vaccine: a content analysis of a representative sample of U.S. newspaper articles. Health Commun 2011 Sep;26(6):491-501. [doi: 10.1080/10410236.2011.556080] [Medline: 21469005]

5. Casciotti DM, Smith KC, Tsui A, Klassen AC. Discussions of adolescent sexuality in news media coverage of the HPV vaccine. J Adolesc 2014 Feb;37(2):133-143 [FREE Full text] [doi: 10.1016/j.adolescence.2013.11.004] [Medline: 24439619]

6. Walker T, Elam-Evans L, Singleton J, Yankey D, Markowitz LE, Fredua B, et al. National, regional, state, and selected local area vaccination coverage among adolescents aged 13-17 years-United States, 2016. MMWR Morb Mortal Wkly Rep 2017 Aug 25;66(33):874-882 [FREE Full text] [doi: 10.15585/mmwr.mm6633a2] [Medline: 28837546]

7. Office of Disease Prevention and Health Promotion. Immunization and Infectious Diseases URL: https://www. healthypeople.gov/2020/topics-objectives/topic/immunization-and-infectious-diseases/objectives?topicId=23 [accessed 2019-02-11] [WebCite Cache ID 767B4Bloc]

8. Darden PM, Thompson DM, Roberts JR, Hale JJ, Pope C, Naifeh M, et al. Reasons for not vaccinating adolescents: National Immunization Survey of Teens, 2008-2010. Pediatrics 2013 Apr;131(4):645-651. [doi: 10.1542/peds.2012-2384] [Medline: $\underline{23509163]}$

9. Gilkey M, McRee AL. Provider communication about HPV vaccination: a systematic review. Hum Vaccin Immunother 2016 Dec 02;12(6):1454-1468 [FREE Full text] [doi: 10.1080/21645515.2015.1129090] [Medline: 26838681]

10. Wong KY, Do YK. Are there socioeconomic disparities in women having discussions on human papillomavirus vaccine with health care providers? BMC Womens Health 2012 Oct 04;12:33 [FREE Full text] [doi: 10.1186/1472-6874-12-33] [Medline: 23033931]

11. Katz I, Bogart L, Fu C, Liu Y, Cox J, Samuels R, et al. Barriers to HPV immunization among blacks and latinos: a qualitative analysis of caregivers, adolescents, and providers. BMC Public Health 2016 Dec 25;16(1):874 [FREE Full text] [doi: 10.1186/s12889-016-3529-4] [Medline: 27558506]

12. Rosenberg K, Mechcatie E. HPV vaccination and sexual behaviors in adolescents and young women. Am J Nurs 2018 Dec;118(4):69. [doi: 10.1097/01.NAJ.0000532080.57125.48] [Medline: 29596260]

13. Reiter PL, Brewer NT, Gottlieb SL, McRee AL, Smith JS. Parents' health beliefs and HPV vaccination of their adolescent daughters. Soc Sci Med 2009 Aug;69(3):475-480. [doi: 10.1016/j.socscimed.2009.05.024] [Medline: 19540642]

14. Jena AB, Goldman DP, Seabury SA. Incidence of sexually transmitted infections after human papillomavirus vaccination among adolescent females. JAMA Intern Med 2015 Apr;175(4):617-623 [FREE Full text] [doi: 10.1001/jamainternmed.2014.7886] [Medline: 25664968]

15. Daley EA, Vamos CA, Zimet GD, Rosberger Z, Thompson EL, Merrell L. The feminization of HPV: reversing gender biases in US human papillomavirus vaccine policy. Am J Public Health 2016 Jun;106(6):983-984. [doi: 10.2105/AJPH.2016.303122] [Medline: 27153015]

16. Wailoo K, Livingston J, Epstein S, Aronowitz R. Three shots at prevention: The HPV vaccine and the politics of medicine's simple solutions: Johns Hopkins University Press; 2010:320 p.

17. Johnson KL, Lin MY, Cabral H, Kazis LE, Katz IT. Variation in human papillomavirus vaccine uptake and acceptability between female and male adolescents and their caregivers. J Community Health 2017 Jun;42(3):522-532 [FREE Full text] [doi: 10.1007/s10900-016-0284-5] [Medline: 27778139]

18. Mourad M, Jetmore T, Jategaonkar A, Moubayed S, Moshier E, Urken M. Epidemiological trends of head and neck cancer in the United States: a SEER population study. J Oral Maxillofac Surg 2017 Dec;75(12):2562-2572 [FREE Full text] [doi: 10.1016/j.joms.2017.05.008] [Medline: 28618252]

19. Abdelmutti N, Hoffman-Goetz L. Risk messages about HPV, cervical cancer, and the HPV vaccine gardasil in North American news magazines. J Cancer Educ 2010 Sep;25(3):451-456. [doi: 10.1007/s13187-010-0087-9] [Medline: 20232189]

20. Smith A, Anderson M. Pew Internet. Washington, DC; 2018 Mar 08. Social media use in 2018 URL: http://www. pewinternet.org/2018/03/01/social-media-use-in-2018/ [accessed 2019-02-11] [WebCite Cache ID 767CkiQXd]

21. Betsch C, Brewer N, Brocard P, Davies P, Gaissmaier W, Haase N, et al. Opportunities and challenges of Web 2.0 for vaccination decisions. Vaccine 2012 May 28;30(25):3727-3733. [doi: 10.1016/j.vaccine.2012.02.025] [Medline: 22365840]

22. McRee AL, Reiter PL, Brewer NT. Parents' internet use for information about HPV vaccine. Vaccine 2012 May 28;30(25):3757-3762 [FREE Full text] [doi: 10.1016/j.vaccine.2011.11.113] [Medline: 22172505]

23. Reddit. 2018. Traffic Statistics URL: https://www.alexa.com/siteinfo/reddit.com [accessed 2019-02-11] [WebCite Cache ID $767 \mathrm{Cvg} 3 \mathrm{Ce}]$

24. Reddit. 2017. URL: https://www.redditinc.com/ [accessed 2019-02-17] [WebCite Cache ID 76GaNbjQN] 
25. Briones R, Nan X, Madden K, Waks L. When vaccines go viral: an analysis of HPV vaccine coverage on YouTube. Health Commun 2012;27(5):478-485. [doi: 10.1080/10410236.2011.610258] [Medline: 22029723]

26. Guidry JP, Carlyle K, Messner M, Jin Y. On pins and needles: how vaccines are portrayed on Pinterest. Vaccine 2015 Sep 22;33(39):5051-5056. [doi: 10.1016/j.vaccine.2015.08.064] [Medline: 26319742]

27. Shapiro G, Surian D, Dunn A, Perry R, Kelaher M. Comparing human papillomavirus vaccine concerns on Twitter: a cross-sectional study of users in Australia, Canada and the UK. BMJ Open 2017 Oct 05;7(10):e016869 [FREE Full text] [doi: 10.1136/bmjopen-2017-016869] [Medline: 28982821]

28. Meacham MC, Paul MJ, Ramo DE. Understanding emerging forms of cannabis use through an online cannabis community: an analysis of relative post volume and subjective highness ratings. Drug Alcohol Depend 2018 Dec 01;188:364-369. [doi: 10.1016/j.drugalcdep.2018.03.041] [Medline: 29883950]

29. Sowles SJ, McLeary M, Optican A, Cahn E, Krauss MJ, Fitzsimmons-Craft EE, et al. A content analysis of an online pro-eating disorder community on Reddit. Body Image 2018 Mar;24:137-144. [doi: 10.1016/j.bodyim.2018.01.001] [Medline: 29414146]

30. Barthel M, Stocking G, Holcomb J, Mitchell A. Pew Research Center. Washington, DC; 2016. Reddit news users more likely to be male, young and digital in their news preferences URL: http://www.journalism.org/2016/02/25/ reddit-news-users-more-likely-to-be-male-young-and-digital-in-their-news-preferences/ [accessed 2019-02-11] [WebCite Cache ID 767DCmEaT]

31. Reddit. 2011. Who in the World is reddit? Results are in... URL: https://redditblog.com/2011/09/12/ who-in-the-world-is-reddit-results-are-in/ [accessed 2019-02-17] [WebCite Cache ID 76GakchqO]

32. Broniatowski DA, Jamison AM, Qi S, AlKulaib L, Chen T, Benton A, et al. Weaponized health communication: Twitter bots and Russian trolls amplify the vaccine debate. Am J Public Health 2018 Oct;108(10):1378-1384. [doi: 10.2105/AJPH.2018.304567] [Medline: 30138075]

33. Pushshift. Learn about big data and social media ingest and analysis URL: https://pushshift.io/using-bigquery-with-reddit-data/ [accessed 2019-02-18] [WebCite Cache ID 76HFiVv01]

34. Graham J, Haidt J, Nosek BA. Liberals and conservatives rely on different sets of moral foundations. J Pers Soc Psychol 2009 May;96(5):1029-1046. [doi: 10.1037/a0015141] [Medline: 19379034]

35. Deerwester S, Dumais ST, Furnas GW, Landauer TK, Harshman R. Indexing by latent semantic analysis. J Am Soc Inf Sci 1990;41(6):391-407. [doi: 10.1002/(SICI)1097-4571(199009)41:6\%3C391::AID-ASI1\%3E3.0.CO;2-9]

36. Blei D, Ng A, Jordan M. Latent Dirichlet Allocation. J Mach Learn Res 2003;3:993-1022 [FREE Full text]

37. Gilbert E. Widespread Underprovision on Reddit. In: Proceedings of the 2013 conference on Computer supported cooperative work.: Association for Computing Machinery; 2013 Presented at: Conference on computer-supported cooperative work and social computing; February 23-27, 2013; San Antonio, Texas p. 803-808.

38. Bednarczyk RA, Davis R, Ault K, Orenstein W, Omer SB. Sexual activity-related outcomes after human papillomavirus vaccination of 11- to 12-year-olds. Pediatrics 2012 Nov;130(5):798-805. [doi: 10.1542/peds.2012-1516] [Medline: 23071201]

39. Mullins TL, Rosenthal SL, Zimet GD, Ding L, Morrow C, Huang B, et al. Human papillomavirus vaccine-related risk perceptions do not predict sexual initiation among young women over 30 months following vaccination. J Adolesc Health 2018 Feb;62(2):164-169 [FREE Full text] [doi: 10.1016/j.jadohealth.2017.09.008] [Medline: 29198772]

40. Krakow M, Rogers B. Collateral damage and critical turning points: public health implications of HPV vaccine news coverage for boys and men in 2011. Health Commun 2016 Dec;31(9):1081-1088. [doi: 10.1080/10410236.2015.1038773] [Medline: 26799666]

41. Ueda Y, Enomoto T, Sekine M, Egawa-Takata T, Morimoto A, Kimura T. Japan's failure to vaccinate girls against human papillomavirus. Am J Obstet Gynecol 2015 Mar;212(3):405-406. [doi: 10.1016/j.ajog.2014.11.037] [Medline: 25434842]

42. Burdette AM, Webb NS, Hill TD, Jokinen-Gordon H. Race-specific trends in HPV vaccinations and provider recommendations: persistent disparities or social progress? Public Health 2017 Jan;142:167-176. [doi: 10.1016/j.puhe.2016.07.009] [Medline: 27592005]

43. Hilton S, Hunt K, Langan M, Bedford H, Petticrew M. Newsprint media representations of the introduction of the HPV vaccination programme for cervical cancer prevention in the UK (2005-2008). Soc Sci Med 2010 Mar;70(6):942-950 [FREE Full text] [doi: 10.1016/j.socscimed.2009.11.027] [Medline: 20064682]

44. Gilkey MB, Magnus BE, Reiter PL, McRee AL, Dempsey AF, Brewer NT. The Vaccination Confidence Scale: a brief measure of parents' vaccination beliefs. Vaccine 2014 Oct 29;32(47):6259-6265 [FREE Full text] [doi: 10.1016/j.vaccine.2014.09.007] [Medline: 25258098]

45. Massey PM, Budenz A, Leader A, Fisher K, Klassen AC, Yom-Tov E. What drives health professionals to tweet about \#HPV vaccine? Identifying strategies for effective communication. Prev Chronic Dis 2018 Dec 22;15:E26 [FREE Full text] [doi: 10.5888/pcd15.170320] [Medline: 29470166]

46. US Food and Drug Administration. FDA approves expanded use of Gardasil 9 to include individuals 27 through 45 years old. 2018. URL: https://www.fda.gov/NewsEvents/Newsroom/PressAnnouncements/ucm622715.htm [accessed 2019-02-11] [WebCite Cache ID 767EXxXhK] 


\author{
Abbreviations \\ ACIP: Advisory Committee on Immunization Practices \\ CDC: Centers for Disease Control and Prevention \\ HCP: health care provider \\ HPV: human papillomavirus \\ LDA: Latent Dirichlet Allocation \\ LSA: Latent Semantic Analysis \\ NIH: National Institutes of Health \\ STI: sexually transmitted infection
}

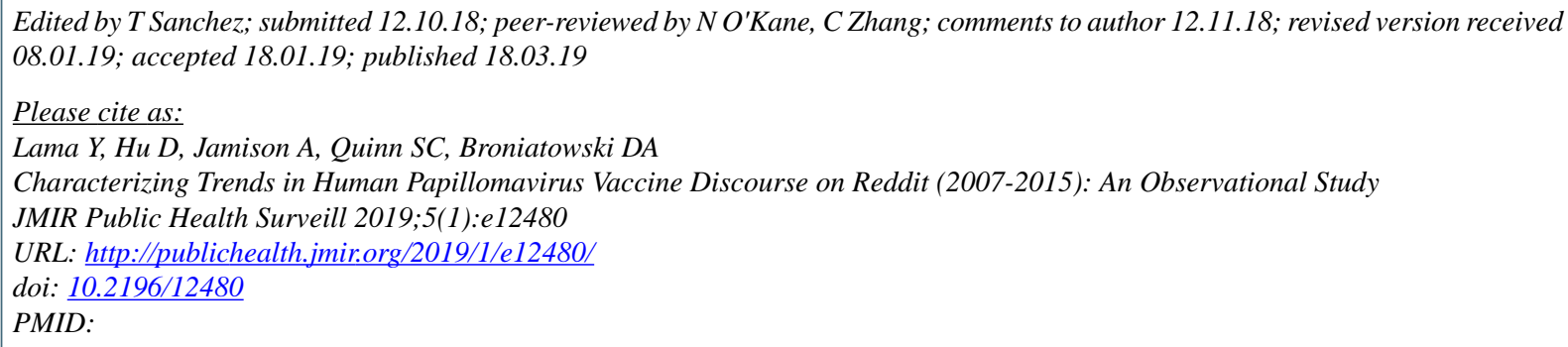

(C) Yuki Lama, Dian Hu, Amelia Jamison, Sandra Crouse Quinn, David A Broniatowski. Originally published in JMIR Public Health and Surveillance (http://publichealth.jmir.org), 18.03.2019. This is an open-access article distributed under the terms of the Creative Commons Attribution License (https://creativecommons.org/licenses/by/4.0/), which permits unrestricted use, distribution, and reproduction in any medium, provided the original work, first published in JMIR Public Health and Surveillance, is properly cited. The complete bibliographic information, a link to the original publication on http://publichealth.jmir.org, as well as this copyright and license information must be included. 